factual stepping stones. The Earth has had its surfaces continuously reshaped by erosion, but on the Moon, for example, a primaeval surface still shows the scars formed in the final stages of mass accretion. Farther afield it might soon be possible to study the planetary systems recently discovered around some of the nearest stars.

\section{CANCER RESEARCH}

\section{Initiation of Tumours}

from a Correspondent

AT the eleventh meeting in the principles of experimental and clinical oncology series in the British Museum (Natural History) on March 20, the speakers were Dr M. Balls (University of East Anglia) and Dr A. M. Neville (Institute of Cancer Research).

Dr Balls, speaking first, set out to be provocative. As a developmental biologist there were many facets of the thoughts of those interested in cancer with which he was unsympathetic. For his part he did not believe that cancer should be thought of as a disease of cells nor of homotypic collections of cells, but a disease of (developmentally) heterotypic cells. His thesis was that it is the changed relationship between heterotypic cells which is most important in the initiation of tumours.

Speaking of differentiation in general, Dr Balls said that nuclear transplantation experiments had established that differentiation did not involve irreversible genomic changes-by inference he did not feel that such changes were a necessary prerequisite of adoption of the malignant state. Furthermore, passage of macromolecules between cells was accepted as an important component of differentiational mechanisms. No cell-including a malignant cellin a metazoan organism should be thought of as an island.

Describing the various classes of cells which could be recognized by the developmental biologist, such as precompetent (not inducible) and competent (responsive to extracellular forces by becoming determined) cells, Dr Balls went on to say that expression of a determined characteristic could be delayed for very many cell generations after the determining event. He therefore found no special mystery in the phenomenon of latency in relation to tumour development. $\mathrm{He}$ indicated that interaction between mesenchymal and epidermal cells could lead to maintenance of a differentiated function in the epidermal cells or even modulation of their behaviour. He exemplified this by the rather atypical reaction which is demonstrable between skin or gizzard mesenchyme and skin epidermis. This last tissue when separated from mesenchyme, tends to undergo end-cell keratinization. When placed in contact with skin mesenchyme it will tend to maintain keratin production and the capacity for replacement of cells, and when placed in contact with gizzard mesenchyme it can produce cilia and mucin. This last modulation is reversible. On the basis of observations such as these Dr Balls felt that it was at least possible to propose that the changes which lead to the production of a malignant cell population probably include changes which are external to the potentially malignant cells.

Dr Neville gave a critical account of tumour index substances, some of which seem to be materials normally produced only, or only in large quantities, during foetal life. He identified as substances of interest in relation to diagnosis or prognosis of tumours, hormones, isozymes, phase specific proteins, $\alpha$-foetoprotein, carcino-embryonic antigen, foetal sulpho-glycoproteins and $\alpha 2-\mathrm{H}$ foetoproteins. All of these substances had their intrinsic interest; none of them seemed on the basis of the present evidence to be unequivocal indicators of malignancy for diagnostic purposes, but many could be used as indices of progress during cancer therapy.

\section{DIPHTHERIA}

\section{What Makes Toxin Tick?}

from our Molecular Biology Correspondent THE work, chiefly of Pappenheimer and his colleagues, in laying bare the mechanism of action of diphtheria toxin is as pretty a piece of what might be called molecular aetiology as would bring tears of pleasure to the eyes of the departed pioneers of bacteriology. The toxin is a protein of molecular weight $60-65,000$, which is progressively cleaved by proteases in the cell extract into pieces of 24,000 (A) and 38,000 (B) that remain bound together by a disulphide bond. The same effect can be achieved, as was shown last year by Gill and Dinius, and by Collier and Kandel, by treatment with trypsin. After reduction, the pieces may be separated, and it is found that the biochemical activity resides in the smaller of the two. This activity is the specific inhibition of eukaryotic translocase, which brings protein synthesis to an instant halt. It achieves this effect by an NAD-dependent ribosylation. The fragment possesses no toxicity in animals, however, which prompts the attractive hypothesis that the other part of the toxin is needed to carry the molecule through the cell membrane and into the cytoplasm, where it can do its work.

Last year Uchida, Gill and Pappenheimer reported the isolation of a mutant form of the toxin from lysogenized bacteria, which possesses no toxicity, and has a molecular weight of 45,000 . Nevertheless it is enzymatically active, and on proteolysis and reduction yields a functional A-fragment. Uchida, Pappenheimer and Harper (Science, 175,901 ; 1972) have now prepared a new mutant of the lysogenic phage, with a missense mutation in the toxin gene, that generates a strain of the diphtheria bacterium with an inactive toxin molecule, which has normal molecular

\title{
Association of Pulsars with Supernova Remnants
}

THE high velocities of pulsars seem to be consistent with the view that they were ejected from supernova explosions. This makes it difficult to determine with certainty definite pulsar-supernova remnant associations, and even in the Crab there remains some doubt about whether or not there is a "runaway" pulsar associated with the system, as well as NP 5032. But M. I. Large and A. E. Vaughan have now established that there is a good likelihood that PSR $1154-62$ is associated with the galactic radio source G296.8-0.3, the Crux supernova remnant (Nature Physical Science, next Monday, April 24).

Observations of the radio source made with the Molonglo Cross (see figure) show the shell structure of the source and its proximity to the pulsar. Although PSR 1154-62 lies outside the shell border, it has a velocity of more than $300 \mathrm{~km} \mathrm{~s}^{-1}$, sufficient to account for its present position, if it was indeed formed with such a velocity, in the Crux supernova.
Furthermore, the age of the remnant $\left(2.5 \times 10^{4} \mathrm{yr}\right)$ is not inconsistent with the presumed age of the pulsar $\left(10^{4}\right.$ to $10^{6} \mathrm{yr}$ ) and it is interesting that, as in the case of the Vela remnant and PSR $0833-45$, the pulsar is adjacent to the brightest region of the shell source. On balance, it seems likely that the juxtaposition of the Crux remnant and PSR 1154-62 on the sky is not a chance occurrence.

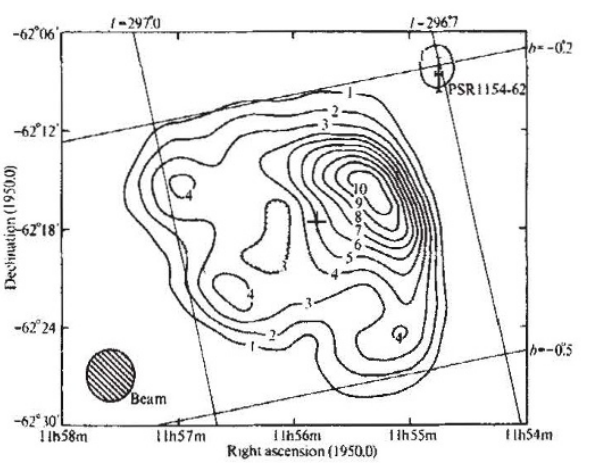

